\title{
Effect of Early Return to Work After Laparoscopic Total Extraperitoneal Hernia Repair: a Retrospective Comparative Cohort Study
}

\section{Ting-En Tai}

Taipei Medical University Hospital

\section{An-Chi Chou}

Taipei Medical University Hospital

Ching-Che Lin

Taipei Medical University Hospital

Chih-Kuan Wu

Taipei Medical University Hospital

Yao-Chou Tsai ( $\nabla$ tsai1970523@yahoo.com.tw)

Taipei Medical University Hospital

\section{Research Article}

Keywords: Hernia repair, Laparoscopic total extraperitoneal, Return to work, Recurrence, Chronic postoperative inguinal pain

Posted Date: June 15th, 2021

DOI: https://doi.org/10.21203/rs.3.rs-585967/v1

License: (c) (i) This work is licensed under a Creative Commons Attribution 4.0 International License. Read Full License 


\section{Abstract}

\section{Background}

This study compares the outcomes of early and late return to work after laparoscopic total extraperitoneal inguinal hernia repair (TEP).

\section{Methods}

Between March 2008 and December 2019, we reviewed 506 cases who underwent laparoendoscopic total extraperitoneal hernia repair (TEP). Of these, 231 cases, who returned to work within one week after surgery, were classified as the early group, and 275 cases, who had no job or returned to work after longer than one week, were classified as the late group. Primary endpoint is inguinal hernia recurrence. Secondary endpoints were post-operative chronic inguinal pain, which is defined as persistent pain 6 months after operation, seroma formation, and modified medical outcome study (MOS) score.

\section{Results}

The two groups had comparable baseline characteristics, except the early group were younger $(51 \pm 13.1$ vs. $58.2 \pm 15.9, p<0.001)$ and had less constipation risk before operation $(10.0 \%$ vs. $18.5 \%, p=0.006)$. The early return to work group did not increase inguinal hernia recurrence rate $(1.7 \%$ versus $2.9 \%, p=0.386)$. Furthermore, the early group is associated with significant less chronic pain (4.8\% versus $11.6 \%$, $p=0.006)$. There is no difference in post-operative seroma formation or MOS scores between early and late group.

\section{Conclusion}

Patients who underwent laparoscopic TEP hernia repair and returned to work within one week did not show increased hernia recurrence rate or complications. Besides, early return to work was associated with significant less chronic pain. Early return to work after TEP repair is safe and feasible. Patients are encouraged to return to work earlier after TEP.

\section{Introduction}

Inguinal hernia repair is a common operation for symptomatic hernia. Laparoscopic total extraperitoneal hernia repair (TEP) is one of the widely used surgical methods. In comparison to open hernia repair, endoscopic hernia repair has advantages of fewer wound infections, fewer hematoma formation, and earlier return to normal activities or work ${ }^{1,2}$. However, early post-operative physical activities are one of the concerns that may increase mesh migration or irritation, which may lead to inguinal hernia recurrence. Intra-abdominal pressure could be increased by physical activity and converted to tensile force ${ }^{3}$. In porcine models, tissue growth into mesh occurred by 2 weeks after laparoscopic placement of hernia prosthesis ${ }^{4}$. The strength of tissue attachment to the mesh increased gradually until 12 weeks after surgery. As a result, the appropriate timing of return to work or activity is important. 
With advances in surgical techniques, the goal of an early return to work and normal leisure activities after minimally invasive surgery is progressing. Although surgeons may recommend patients resume leisure activities as soon as the patient can tolerate it, patients cannot choose the physical loading or duration if returning to work. European Hernia Society guidelines give a grade $\mathrm{C}$ recommendation that patients are free to resume activity after inguinal hernia repair without mentioning specific operation method, except heavy weight lifting work ${ }^{1}$. Until now, there is no global consensus in the timing of returning to work for patients who underwent laparoscopic hernia repair. Therefore, we investigated the outcomes in patients of early and late return to work after laparoscopic total extraperitoneal (TEP) inguinal herniorrhaphy.

\section{Methods}

\subsection{Patient selection}

This is a retrospective study that analyzed the clinical information extracted from a prospectively collected database. From March 2008 to December 2019, 885 patients who underwent elective laparoscopic TEP inguinal hernia repair were enrolled in this database. Patients who had concurrent major abdominal surgery or emergent operations were not enrolled. All operations were performed by a single experienced surgeon, who had performed more than 200 laparoscopic TEP surgeries before initiation of the database collection. Patients' baseline demographics and perioperative outcomes were prospectively collected. Inform consent was obtained from all subjects or, if subjects are under 18 , from a parent or legal guardian. The study protocol was approved by the Institutional Review Board of Buddhist Taipei Tzu-Chi General Hospital (IRB code: 08-X-040). Moreover, the study followed STROCSS guideline ${ }^{5}$ and all methods were performed in accordance with the relevant guidelines and regulations or Declaration of Helsinki. ${ }^{6}$

The aim of this study is to analyze the outcome differences between patients returning to work early and late after laparoscopic TEP repair. Therefore, patients who had an intra-operative conversion to transabdominal preperitoneal (TAPP) method, follow-up of less than 6 months, and lack of information of returning to work were excluded for the final analysis (Fig. 1). According to the timing of returning to work, 231 patients were in the early group (returned to work within one week after surgery), and 275 patients were in the late group (returned to work more than 1 week after surgery or unemployed). The physical loading of work types was classified into three levels (light: sedentary and lifting less than 5 kilograms; medium: lifting 5 to 10 kilograms; heavy: lifting more than 10 kilograms).

\subsection{Post operation follow-up and assessment}

All patients were followed standardized post-operative care, including early oral intake and early ambulation. There were no post-operative physical activities restriction. Patients were encouraged to resume activities as it can be tolerated. Painkillers were administered according to patients' need. Acute post-operative pain was evaluated by Visual Analog Scale (VAS) rulers with 0-100 on post-operative day 1 
and day 7. We accessed patients for pain, complications, and activity level at outpatient clinic at postoperative 1 week, 3 months and 6 months. The timing of return to work was recorded by questionnaire. The primary outcome is inguinal hernia recurrence rate. The secondary outcomes are chronic inguinal pain, seroma formation, and activity level. Chronic inguinal pain is defined by persistent pain more than 6 months after surgery and was assessed by an independent trained nursing staff at outpatient clinic or via telephone interview. We evaluated activity level by modified Medical Outcome Score (MOS; 3-12 items; scores range from 3 to 30 ) on post-operative day 7 and 3 months (Supplement Table 1.).

\subsection{Statistical analysis}

Statistical analyses were performed with Pearson Chi square for category variables. Continuous variables were tested with Kolmogorov-Smirnov test for normality and compared with $\mathrm{t}$ test or Mann-Whitney $\mathrm{U}$ test. Multivariate analyses using logistic or linear regression were carried out and adjusted for sex, age, $\mathrm{BMI}$, pain before surgery, mesh fixation methods, mesh types, and operation methods. All tests were twotailed and considered as statistically significant as $p<0.05$. Statistical analyses were performed with IBM SPSS statistical software version 26 for Windows (IBM Corp., New York, USA).

\section{Results}

The baseline demographic data is presented in Table 1, and the two groups had comparable characteristics in terms of sex, body mass index, comorbidity, initial symptoms, recurrent hernia, and laterality. The early group was younger than the late group ( $51.3 \pm 13.1$ versus $58.2 \pm 15.9$ years old, $p<$ 0.001). Besides, the distribution of work types was significant different among the two groups, with more light work types in the early group. The early group had less risk factors of benign prostate obstruction (19.5\% versus $28.7 \%, p=0.016)$ and constipation $(10.0 \%$ versus $18.5 \%, p=0.006)$ compared to the late group.

There was no significant difference regarding operation method, hernia type, mesh material and fixation methods, hernia defect size, and operation time between early and late groups (Table 2 ). The early group had a shorter hospital stay than the late group $(23.3 \pm 9.9$ versus $25.2 \pm 12.9$ hours, $p=0.03)$ (Table 3 ). For the primary outcome, there is no significant different inguinal hernia recurrence rate between the two groups. After adjusted for work types and potential confounding factors, the early groups showed lower odds ratio compared to the late group without statistically significance (Odds ratio: $0.548,95 \%$ confidence interval: $0.142,2.122, p=0.384$ ) (Table 4). In view of complications, the two groups had similar seroma formation, wound infection, and epididymitis, except that the early group had less acute urine retention $(3.9 \%$ versus $8.7 \%, p=0.028)$. On the post operation day 1 , the early group had significant higher VAS pain scores at rest $(1.8 \pm 1.8$ versus $1.4 \pm 1.7, p=0.018)$. After adjustment with multivariable linear regression, there were no significant differences in VAS pain scores at rest on post operation day 1 between the early and late groups (Table 4).

Nevertheless, the early group had significantly less chronic pain compared to the late group (4.8\% versus $11.6 \%, p=0.006)$. Multivariable logistic regression also showed the early group is a protective factor of 
chronic pain after adjusting for potential confounding factors (Odds ratio: $0.319 ; 95 \%$ confidence interval: $0.151,0.674 ; p=0.003$ ). The outcomes of modified Medical Outcome Score (MOS) revealed significant higher scores in the early group on post-operative day $7(27.10 \pm 1.60$ versus $26.51 \pm 2.02, p=0.001)$ but comparable scores on post-operative day 1 and at month 3 .

\section{Discussion}

This is the first comprehensive study exploring the impact of going back to work early in the laparoscopic hernia repair era. Our study demonstrated that going back to work within one week after laparoscopic TEP hernia repair dose not increase inguinal hernia recurrence and seroma formation. In addition, patients going back to work early had significantly less chronic inguinal pain. Early and late groups had similar functional outcomes at three months after operation. These findings suggest early return to work is feasible and safe for patients who underwent laparoscopic TEP.

In the open hernia repair era, early return to work was an important convalescence goal of operation. It was associated with socioeconomic advantages, including decreasing loss of productivity and earning of workers ${ }^{7}$. A review made by Forbes et al. recommended that patients could return to work 1 to 2 weeks after open hernia repair if little or no lifting was involved or 6-8 weeks if lifting more than 10 kilograms $^{8}$. The Hernia Surge Group recommended that patients could resume work or activity within 3-5 days after elective laparoscopic or open hernia repair (upgraded to strong recommendation by panel discussion) ${ }^{9}$. Furthermore, a recent study investigated the determinants of short convalescence after laparoscopic hernia repair showed a median of 5 days of convalescence from work ${ }^{10}$. In our study, we classified patients into the early group if resuming work within 7 days after surgery and the results showed similar recurrence and complication rate compared to the late group. Based on our findings, patients are free to go back to work early after laparoscopic TEP hernia repair.

One of the important considerations of early return to work is inguinal hernia recurrence. Our study revealed that returning to work within one week did not increase inguinal hernia recurrence. From the experience of open hernia, Taylor reported that patients had comparable hernia recurrence rates between early (full work duties at 21 days after surgery) and late (full work duties at 3 months) return to work ${ }^{11}$. Another large-scale open hernia repair study by Bay-Neilsen et al. presented no significant difference in recurrence rate between non-restrictive $(n=1059)$ and restrictive $(n=1306)$ convalescence recommendation groups $(0.7 \%$ versus $1.6 \%, p=0.186)$, in which median time off work after surgery was 7 days in non-restrictive recommendation groups ${ }^{12}$. As compared to open hernia repair, laparoscopic TEP hernia repair offers an earlier return to work ( 6.4 days versus 11.5 days, $p<0.001)^{13}$. Therefore, it is reasonable to define early return to work as within one week after surgery in our study. The early and late groups had comparable hernia recurrence rates $(1.7 \%$ versus $2.9 \%, p=0.386)$ after follow-up of more than 6 months (Table 3 ). From the results of our study, early return to work did not increase the incidence of inguinal hernia recurrence. 
Chronic postoperative inguinal pain is a bothersome complication for patients. A review by Poobalan et al. showed that patients who underwent laparoscopic hernia repair had great variations in incidence of chronic pain $(0.8-29 \%)^{14}$. Our study revealed the incidence of chronic pain is $4.8 \%$ and $11.6 \%$ in the early and late groups, respectively $(p=0.006)$. A younger age was one of the risk factors of chronic postoperative inguinal pain in previous studies ${ }^{15-17}$. In our study, the early group was younger than the late group. After adjusting for age, multivariate regression revealed early return to work had significant less chance of developing chronic pain than the late group. Persistent neuralgia resulting from intraoperative neural injury and neuron entrapment could be the main reason of chronic pain ${ }^{18-21}$. Theoretically, early return to work is associated with more early postoperative physical activity. Physical

activity can stimulate blood circulation and angiogenesis, which is beneficial to wound healing $22-24$. Early activity may stimulate tissue regrowth and further prevent nerve irritation by mesh, which in turn decreases chronic pain. The present study demonstrates that returning to work within one week after laparoscopic TEP hernia repair is associated with significant less chronic inguinal pain than those returning more than one week or being unemployed. Based on our findings and references, we recommend patients return to work within one week after laparoscopic TEP hernia repair owing to less chronic inguinal pain.

Post-operative urine retention is one of the common complications after laparoscopic hernia repair. Previous studies identified some risk factors, including age, narcotic medication used, and history of benign prostate hyperplasia ${ }^{25,26}$. Patel et al. reported that patients older than 50 -years-old were associated with post-operative urine retention (odds ratio $3.0, p=0.01)^{25}$. In our study, the late group was older ( 51.3 versus $58.2, p<0.001)$ and had more benign prostate obstruction (BPO) at presentation $(19.5 \%$ versus $28.7 \%, p=0.016)$ (Table 1 ). Although the two groups had comparable post-operative narcotic use (Table 3), more patients with BPO had post-operative urine retention in the late group (3.9\% versus $8.7 \%, p=0.028)$. The way to prevent post-operative urine retention is an important issue warranting further study.

There are some limitations in our study. First, this is a retrospective cohort study. Further prospective investigation with measurable post-operative activity is warranted. Second, the two groups had different distributions in physical loading of work. Limited evidence is available for convalescence from light to heavy work after TEP repair. In addition to work, leisure activity is also an important convalescence factor, and this was not analyzed in the current study.

\section{Conclusion}

In conclusion, our study found that return to work within one week after laparoscopic TEP hernia repair did not increase hernia recurrence rates. Furthermore, patients early return to work are associated with significantly less chronic inguinal pain. Postoperative seroma formation and complication rates are comparable between groups. Therefore, we recommend that patients should return to work early within one week after laparoscopic TEP hernia repair. 


\section{Declarations}

\section{Acknowledgments}

We thank Simon Hall for language editing.

\section{Author contributions}

T.E.T. wrote the main manuscript. T.E.T, A.C.C., C.C.L. and C.K.W. collected and analyzed the data. Y.C.T. constructed the study and produced the final manuscript. All authors reviewed the manuscript.

\section{Funding}

This research received no specific grant from any funding agency in the public, commercial, or not-forprofit sectors.

\section{Competing Interests}

The authors declare no competing interest

\section{References}

1. Simons, M. P. et al. European Hernia Society guidelines on the treatment of inguinal hernia in adult patients. Hernia : the journal of hernias and abdominal wall surgery 13, 343-403, doi:10.1007/s10029-009-0529-7 (2009).

2. McCormack, K., Scott, N., Go, P. M., Ross, S. J. \& Grant, A. Laparoscopic techniques versus open techniques for inguinal hernia repair. Cochrane Database of Systematic Reviews, doi:10.1002/14651858.CD001785 (2003).

3. Konerding, M. A. et al. Maximum forces acting on the abdominal wall: experimental validation of a theoretical modeling in a human cadaver study. Med Eng Phys 33, 789-792, doi:10.1016/j.medengphy.2011.01.010 (2011).

4. Majercik, S., Tsikitis, V. \& lannitti, D. A. Strength of tissue attachment to mesh after ventral hernia repair with synthetic composite mesh in a porcine model. Surg Endosc 20, 1671-1674, doi:10.1007/s00464-005-0660-1 (2006).

5. Agha, R. et al. STROCSS 2019 Guideline: Strengthening the reporting of cohort studies in surgery. Int J Surg 72, 156-165, doi:10.1016/j.ijsu.2019.11.002 (2019).

6. World Medical Association Declaration of Helsinki. Ethical principles for medical research involving human subjects. Bull World Health Organ 79, 373-374 (2001).

7. Bourke, J. B. \& Taylor, M. The clinical and economic effects of early return to work after elective inguinal hernia repair. Br J Surg 65, 728-731, doi:10.1002/bjs.1800651016 (1978).

8. Forbes, J., Fry, N., Hwang, H. \& Karimuddin, A. A. Timing of return to work after hernia repair: Recommendations based on a literature review. British Columbia Medical Journal 54 (2012). 
9. International guidelines for groin hernia management. Hernia 22, 1-165, doi:10.1007/s10029-0171668-x (2018).

10. Tolver, M. A. et al. Determinants of a short convalescence after laparoscopic transabdominal preperitoneal inguinal hernia repair. Surgery 151, 556-563, doi:10.1016/j.surg.2011.08.020 (2012).

11. Taylor, E. W. \& Dewar, E. P. Early return to work after repair of a unilateral inguinal hernia. Br J Surg 70, 599-600, doi:10.1002/bjs.1800701010 (1983).

12. Bay-Nielsen, M. et al. Convalescence after inguinal herniorrhaphy. Br J Surg 91, 362-367, doi:10.1002/bjs.4437 (2004).

13. Savarise, M. T., Simpson, J. P., Moore, J. M. \& Leis, V. M. Improved functional outcome and more rapid return to normal activity following laparoscopic hernia repair. Surg Endosc 15, 574-578, doi:10.1007/s004640080039 (2001).

14. Poobalan, A. S. et al. A review of chronic pain after inguinal herniorrhaphy. Clin J Pain 19, 48-54, doi:10.1097/00002508-200301000-00006 (2003).

15. Aasvang, E. \& Kehlet, H. Chronic postoperative pain: the case of inguinal herniorrhaphy. Br J Anaesth 95, 69-76, doi:10.1093/bja/aei019 (2005).

16. Lo, C. W., Chen, Y. T., Jaw, F. S., Yu, C. C. \& Tsai, Y. C. Predictive factors of post-laparoscopic inguinal hernia acute and chronic pain: prospective follow-up of 807 patients from a single experienced surgeon. Surg Endosc, doi:10.1007/s00464-020-07373-2 (2020).

17. Langeveld, H. R. et al. Prognostic value of age for chronic postoperative inguinal pain. Hernia 19, 549-555, doi:10.1007/s10029-014-1282-0 (2015).

18. Eubanks, S. et al. Meralgia paresthetica: a complication of laparoscopic herniorrhaphy. Surg Laparosc Endosc 3, 381-385 (1993).

19. Rosen, A. \& Halevy, A. Anatomical basis for nerve injury during laparoscopic hernia repair. Surg Laparosc Endosc 7, 469-471 (1997).

20. Stark, E., Oestreich, K., Wendl, K., Rumstadt, B. \& Hagmüller, E. Nerve irritation after laparoscopic hernia repair. Surg Endosc 13, 878-881, doi:10.1007/s004649901124 (1999).

21. Seid, A. S. \& Amos, E. Entrapment neuropathy in laparoscopic herniorrhaphy. Surg Endosc 8, 10501053, doi:10.1007/bf00705717 (1994).

22. Wainwright, T. W. \& Burgess, L. in Enhanced Recovery After Surgery: A Complete Guide to Optimizing Outcomes (eds Olle Ljungqvist, Nader K. Francis, \& Richard D. Urman) 211-218 (Springer International Publishing, 2020).

23. Yuliana, Y., Johan, A. \& Rochana, N. in International Nursing Conference on Chronic Diseases Management. 246-250.

24. Shakil-ur-Rehman, S., Sheikh, S. I. \& Danish, K. F. The role of early mobilization in the prevention of post operative wound infection after lower extremity orthopedic surgeries. Journal of Islamic International Medical College 7, 63-66 (2015). 
25. Patel, J. A., Kaufman, A. S., Howard, R. S., Rodriguez, C. J. \& Jessie, E. M. Risk factors for urinary retention after laparoscopic inguinal hernia repairs. Surg Endosc 29, 3140-3145, doi:10.1007/s00464-014-4039-z (2015).

26. Sivasankaran, M. V., Pham, T. \& Divino, C. M. Incidence and risk factors for urinary retention following laparoscopic inguinal hernia repair. Am J Surg 207, 288-292, doi:10.1016/j.amjsurg.2013.06.005 (2014).

\section{Tables}

Table 1. Baseline patient characteristics 
Return to work

\begin{tabular}{|c|c|c|c|}
\hline & Early $(n=231)$ & Late $(n=275)$ & $p$ value \\
\hline Sex, male $(n, \%)$ & $211(91.3 \%)$ & $248(90.2 \%)$ & 0.654 \\
\hline Age (Mean+SD) & $51.3+13.1$ & $58.2+15.9$ & $<0.001 *$ \\
\hline BMI (Mean+SD) & $24.2+3.2$ & $23.6+3.1$ & 0.058 \\
\hline \multicolumn{4}{|l|}{ Risk factor } \\
\hline Weightlifting & $50(21.6 \%)$ & $65(23.6 \%)$ & 0.594 \\
\hline COPD & $18(7.8 \%)$ & $24(8.7 \%)$ & 0.704 \\
\hline BPO & 45 (19.5\%) & $79(28.7 \%)$ & $0.016^{*}$ \\
\hline Constipation & $23(10.0 \%)$ & $51(18.5 \%)$ & $0.006^{*}$ \\
\hline \multicolumn{4}{|l|}{ Comorbidity } \\
\hline DM & $15(6.5 \%)$ & $31(11.3 \%)$ & 0.063 \\
\hline HTN & $56(24.2 \%)$ & $72(26.2 \%)$ & 0.617 \\
\hline CAD & $8(3.5 \%)$ & $15(5.5 \%)$ & 0.284 \\
\hline CVA & $2(1.0 \%)$ & $7(2.5 \%)$ & 0.191 \\
\hline \multicolumn{4}{|l|}{ Initial symptoms } \\
\hline Pain & $85(36.8 \%)$ & $115(41.8 \%)$ & 0.250 \\
\hline Bulging & $225(97.4 \%)$ & $270(98.2 \%)$ & 0.549 \\
\hline Recurrent & $28(12.1 \%)$ & $33(12.0 \%)$ & 0.967 \\
\hline \multicolumn{4}{|c|}{ Clinical hernia side } \\
\hline Left & 78 (33.8\%) & $80(29.1 \%)$ & 0.335 \\
\hline Right & 84.(36.4\%) & $117(42.5 \%)$ & \\
\hline Bilateral & $69(29.9 \%)$ & $78(28.4 \%)$ & \\
\hline \multicolumn{4}{|l|}{ Work type } \\
\hline Light & $195(84.4 \%)$ & $133(68.2 \%)$ & $0.001 *$ \\
\hline Medium & $20 . .(8.7 \%)$ & $32(16.4 \%)$ & \\
\hline Heavy & 14. $(6.1 \%)$ & $21(10.8 \%)$ & \\
\hline NA & 2. $(0.9 \%)$ & $9 \quad(4.6 \%)$ & \\
\hline No Job & 0 & 80 & \\
\hline
\end{tabular}


*: $p<0.05$; BMI: body mass index; COPD: chronic obstructive pulmonary disease; BPO: benign prostate obstruction; DM: diabetes mellitus; HTN: hypertension; CAD: coronary artery disease; CVA:

cerebrovascular accident; NA: non-available

Table 2. Intraoperative parameters 
Return to work

Early $(\mathbf{n}=\mathbf{2 3 1}) \quad$ Late $(\mathrm{n}=\mathbf{2 7 5}) \quad p$ value

Operation method

\begin{tabular}{llll}
\hline TEP & $54(23.4 \%)$ & $85(30.9 \%)$ & 0.059 \\
\hline LESS TEP & $177(76.6 \%)$ & $190(69.1 \%)$ & \\
\hline Hernia type & & & \\
\hline Indirect & $136(58.9 \%)$ & $157(57.1 \%)$ & 0.598 \\
\hline Direct & $59(25.5 \%)$ & $82(29.8 \%)$ & \\
\hline Femoral & $1(0.4 \%)$ & $3(1.1 \%)$ & \\
\hline Mixed & $31(13.4 \%)$ & $32(11.6 \%)$ & \\
\hline Mesh material & & & \\
\hline Light weight & $130(56.3 \%)$ & $147(53.5 \%)$ & 0.369 \\
\hline Heavy weight & $100(43.3 \%)$ & $125(45.5 \%)$ & \\
\hline Surgisis & $0(0 \%)$ & $2(0.7 \%)$ & 0.519 \\
\hline Mesh fixation & & & \\
\hline Tack & $202(87.4 \%)$ & $233(84.7 \%)$ & \\
\hline Glue & $29(12.6 \%)$ & $40(14.5 \%)$ & \\
\hline None & $0(0 \%)$ & $1(0.4 \%)$ & \\
\hline Hernia defect size & & & \\
\hline$<1.5$ cm & $10(10.3 \%)$ & $10(3.6 \%)$ & \\
\hline $1.5-3$ cm & $54(55.7 \%)$ & $85(61.6 \%)$ & \\
\hline$>3$ cm & $33(34.0 \%)$ & $43(31.2 \%)$ & \\
\hline Operation time (mins) $\#$ & $60.0(48.0,75.0)$ & $60.0(47.0,75.0)$ & 0.3406 \\
\hline Peritoneal tear & $28(12.1 \%)$ & $42(15.3 \%)$ & \\
\hline \#median, IQR & & & \\
\hline
\end{tabular}

Table 3. Post-operative outcomes 
Return to work

\begin{tabular}{|c|c|c|c|}
\hline & Early $(n=231)$ & Late $(n=275)$ & $p$ value \\
\hline Hospital stay (hr) & $23.3 \pm 9.9$ & $25.5 \pm 12.9$ & $0.030 *$ \\
\hline Morphine equivalent dose (mg/kg) & $0.10552 \pm 0.90414$ & $0.09095 \pm 0.72839$ & 0.842 \\
\hline \multicolumn{4}{|l|}{ Complications } \\
\hline Seroma & $31(13.4 \%)$ & $37(13.5 \%)$ & 0.991 \\
\hline AUR & $9(3.9 \%)$ & $24(8.7 \%)$ & $0.028^{*}$ \\
\hline Wound infection & $0(0 \%)$ & $2(0.7 \%)$ & 0.503 \\
\hline UTI & $4(1.7 \%)$ & $3(1.0 \%)$ & 0.708 \\
\hline Epididymitis & $2(0.9 \%)$ & $2(0.7 \%)$ & 1.000 \\
\hline Recurrence & $4(1.7 \%)$ & $8(2.9 \%)$ & 0.386 \\
\hline Chronic pain & $11(4.8 \%)$ & $32(11.6 \%)$ & $0.006^{*}$ \\
\hline \multicolumn{4}{|l|}{ VAS for pain } \\
\hline Cough at ward & $5.5 \pm 2.1$ & $5.1 \pm 2.9$ & 0.109 \\
\hline Cough on POD1 & $4.0 \pm 2.0$ & $3.6 \pm 2.3$ & 0.059 \\
\hline Rest at ward & $3.3 \pm 2.5$ & $3.2 \pm 2.7$ & 0.591 \\
\hline Rest on POD1 & $1.8 \pm 1.8$ & $1.4 \pm 1.7$ & $0.018^{*}$ \\
\hline \multicolumn{4}{|l|}{ Modified medical outcome score } \\
\hline MOS POD1 & $21.67 \pm 4.24$ & $21.14 \pm 4.16$ & 0.176 \\
\hline MOS POD7 & $27.10 \pm 1.60$ & $26.51 \pm 2.02$ & $0.001^{*}$ \\
\hline MOS POM3 & $29.87 \pm 0.47$ & $29.08 \pm 0.84$ & 0.274 \\
\hline Follow up (months)\# & $11.0(6.0,32.0)$ & $12.0(6.0,32.0)$ & 0.860 \\
\hline
\end{tabular}

Table 4. Multivariable regression analysis of visual analog scale (VAS) pain scores, modified medical outcome study (MOS) scores, chronic pain, seroma, and recurrence 


\section{Return to work}

\section{$<1$ week}

\begin{tabular}{llll}
\hline & $\beta$ & $95 \%$ Confidence interval & $p$ value \\
\hline VAS/Cough/ward & -0.091 & $(-0.613,0.431)$ & 0.731 \\
\hline VAS/Cough/POD1 & 0.094 & $(-0.339,0.527)$ & 0.668 \\
\hline VAS/rest/ward & -0.152 & $(-0.653,0.349)$ & 0.551 \\
\hline VAS/rest/POD1 & 0.169 & $(-0.177,0.516)$ & 0.337 \\
\hline MOS POD1 & 0.948 & $(0.125,1.770)$ & $0.024^{\star}$ \\
\hline MOS POD7 & 0.529 & $(0.145,0.912)$ & $0.007^{*}$ \\
\hline MOS POM3 & 0.106 & $(-0.041,0.254)$ & 0.158 \\
\hline & $0 R$ & & $0.001^{\star}$ \\
\hline Chronic pain & 0.283 & $(0.132,0.601)$ & 0.954 \\
\hline Seroma & 1.018 & $(0.552,1.878)$ & 0.384
\end{tabular}

\#adjuster for sex. Age, BMI, pain before operation, mesh fixation method, mesh type, operation method, and work type; VAS: Visual analog scale; POD: post-operative day; POM: post-operative month

\section{Figures}




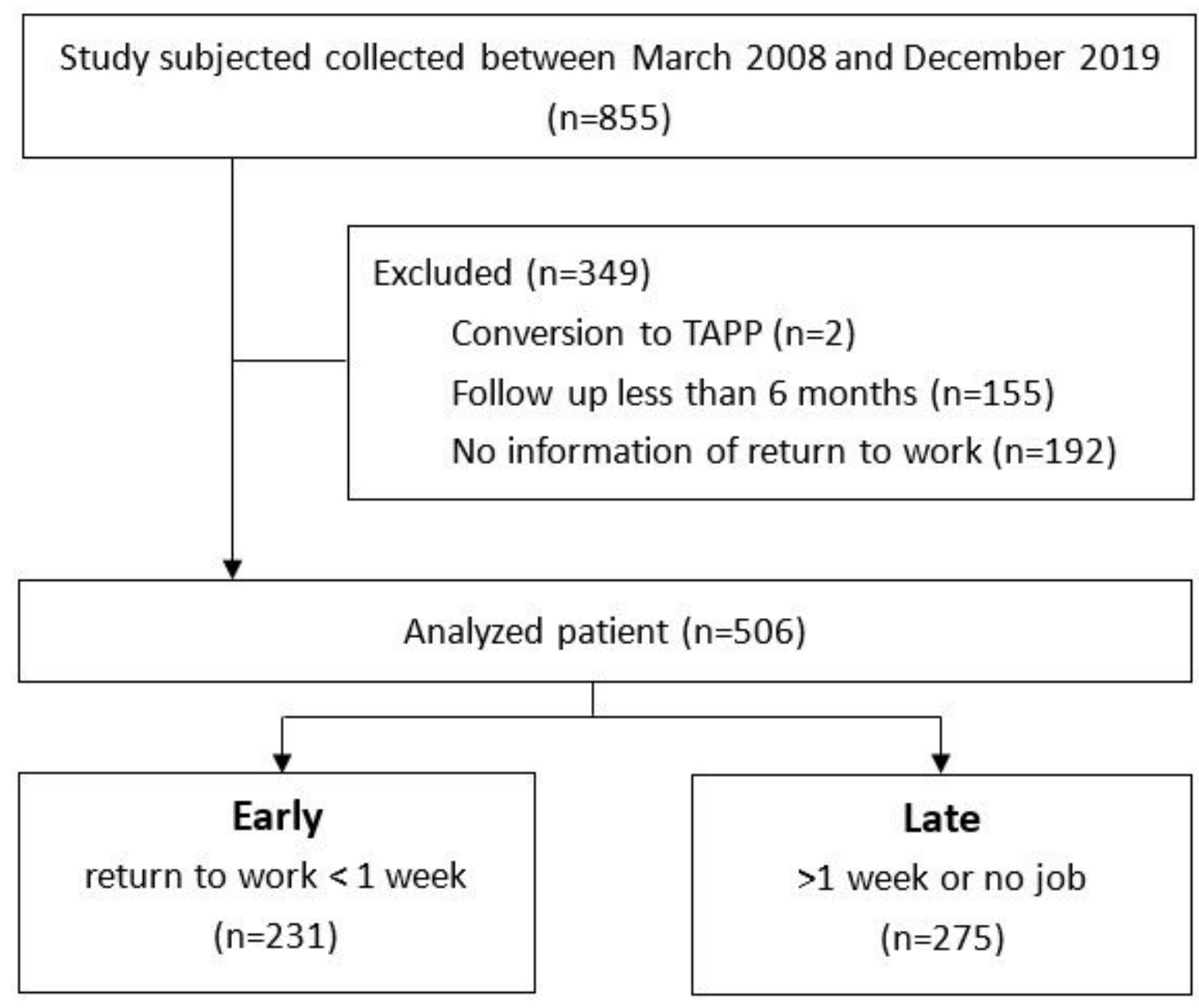

Figure 1

Flowchart of patient inclusion

\section{Supplementary Files}

This is a list of supplementary files associated with this preprint. Click to download.

- Supplementarytable1.docx 